\title{
Optimized Improvement Design of KYN28 Switchgear Based On The Finite Element Model
}

\author{
Jiucheng $\mathrm{Xu} *$ \\ School of Business \\ Hohai University \\ Nanjing, China \\ 744655592@qq.com \\ * Corresponding Author
}

\author{
Renxiang Shen \\ School of Mechanical and Electrical Engineering \\ Hohai University \\ Nanjing, China \\ 328162161@qq.com
}

\begin{abstract}
Nowadays 12kv medium voltage switchgear equipment is widely used in city power supply and distribution system. Combined with the characteristics of KYN28 switchgear, making theoretical analysis with Solidworks software and ANSYS based on the finite element model,the body strength of switchgear is improved and optimized,providing theoretical basis for the entity manufacturing of switchgear, providing theoretical basis for the physical design of switchgear.
\end{abstract}

Keywords- KYN28 switchgear; finite element method;optimized improvement styling; Solidworks;ANSYS

\section{INTRODUCTION}

Nowadays, $12 \mathrm{kv}$ medium voltage switchgear equipment is widely used in city power supply and distribution system, KYN series which are the most representative occupy the biggest market share ${ }^{[1]}$.Switch equipment internal arc fault is a kind of frequent disastrous accidents, the personal and equipment safety is direct threatened. Finite element method is a numerical calculation method of solving the mathematical and a powerful numerical tool of solving engineering problems. Because flexible, fast and effective of this method, it has been widely used in many general fields and practical engineering problems. The finite element analysis can be divided into three stages, pre and after processing, the processing ${ }^{[2-4]}$. Pretreatment is establishing a finite element model and completing unit grid; Post-processing is collecting and processing results of the analysis, making the user extract information simply and understand the calculation results ${ }^{[5]}$.

\section{STRUCTURE ANALYSIS OF SWITCHGEAR'S HOLDER}

\section{A. plank thickness analysis}

The paper accepts the critical tensile stress criterion

$$
\sigma \geq \sigma_{d} \text { (Rinehan1952) }
$$

In formula (1), $\sigma_{d}$ is the fracture threshold or the fracture critical stress, $\mathrm{Gpa}^{[6]}$.

In ANSYS, by establishing a finite element model, define the constraint boundary conditions (according to the actual situation, completely fixed constraints around the plank), using the critical tensile stress criterion of strength theory, according to the value of, estimating the maximum instantaneous pressure that sheet could withstand ${ }^{[7]}$.Safety coefficient is 1.4,the maximum principal stress for the moment is $[\sigma]=\sigma_{d} / 1.4=1.11 \mathrm{Gpa}$. The backdoor plank of cable chamber is relatively large impacted, so select the shell thickness according to internal and external pressure difference of the cable chamber's door,all completely fixed constraint around the plank, choose Solid 10node 187 unit type, applied load type for surface load, size for 223700 pa.

Respectively choose $2 \mathrm{~mm}$ and $3 \mathrm{~mm}$ thick of Q235A steel plate, the principal stress analysis of the first strength theory is shown in Figure 1 and 2.

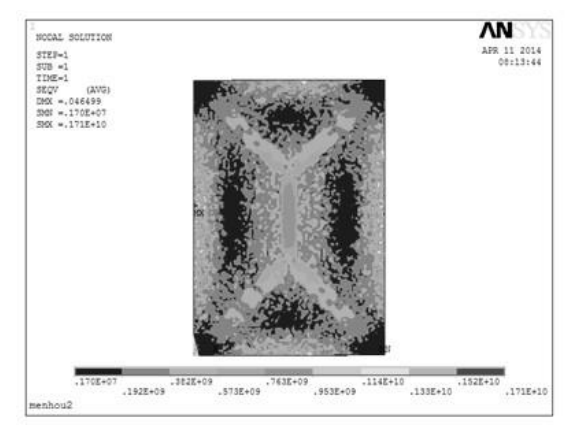

Figure 1. Stress distribution of $2 \mathrm{~mm}$ thick steel plate

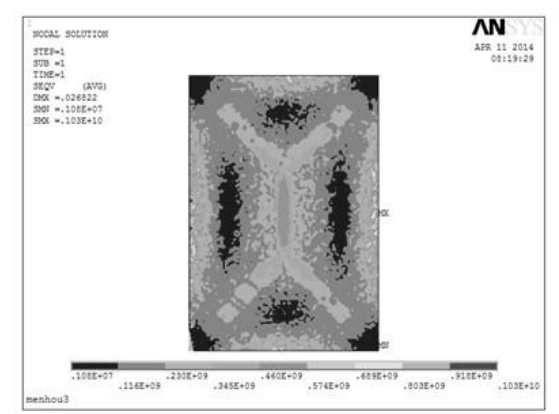

Figure 2. Stress distribution of $2 \mathrm{~mm}$ thick steel plate 


\section{B. The analysis of the circuit breaker chamber's door plank}

Dividing mesh by uniform grid density, choose Solid 10 node 187 unit type, unit length is defined as a $10 \mathrm{~mm}$, as shown in Figure 3.Three threaded hole of door plank and six hinge holes are completely fixed constraint, exert load type is surface load and the size is 177400 pa. Figure 4 shows that before it is torn the maximal displacement deformation of the circuit breaker room door is $40.52 \mathrm{~cm}$, displacement deformation range is larger; The displacement deformation of the right side 1 and the left side 2 on panel is within the scope of $3 \mathrm{~mm}$ which satisfy the requirements; The displacement deformation of the upper side 3 and the lower side 4 on panel is beyond the scope of $6 \mathrm{~mm}$, which doesn't meet the safety requirements, so the gas may emit above and below the door easily and hurt people.

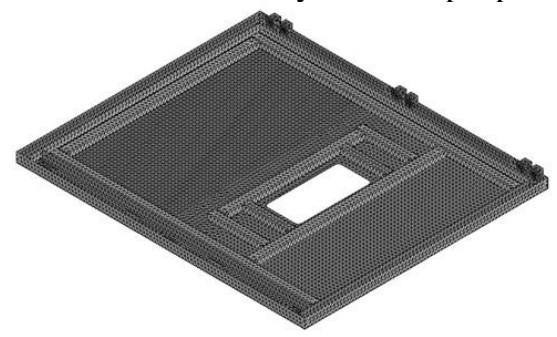

Figure 3. The mesh generation of circuit breaker chamber's door

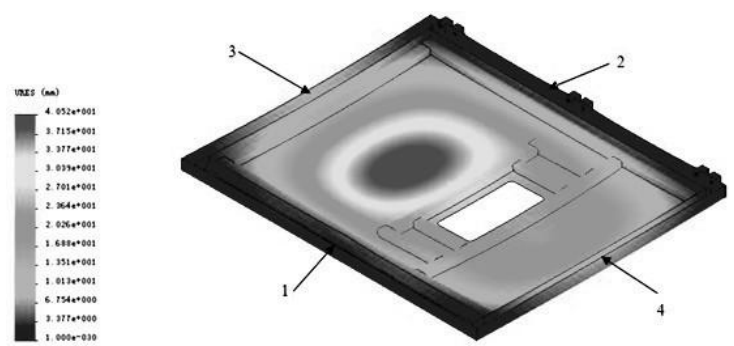

Figure 4. The deformation results of circuit breaker chamber's door

\section{The analysis of the cable room's door plank}

Dividing mesh by uniform grid density, choose Solid 10 node 187 unit type, unit length is defined as a $10 \mathrm{~mm}$, as shown in Figure 5. Four threaded hole of door plank and eight hinge holes are completely fixed constraint, exert load type is surface load and the size is 223700 pa.The figure 6 shows that before it is torn the maximal displacement deformation of the cable room door is 28.56 $\mathrm{cm}$, displacement deformation range is larger;The displacement deformation of the right side 1 and the left side 2 on panel is within the scope of $3 \mathrm{~mm}$ which satisfy the requirements; The displacement deformation of the upper side 3 and the lower side 4 on panel is beyond the scope of $7 \mathrm{~mm}$, which doesn't meet the safety requirements, so the gas may emit above and below the door easily and hurt people.

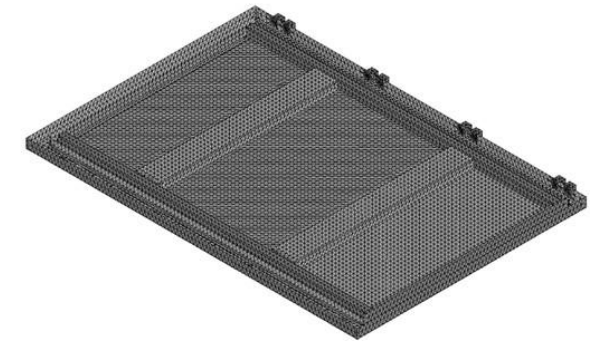

Figure 5. The mesh generation of circuit breaker chamber door

䇎

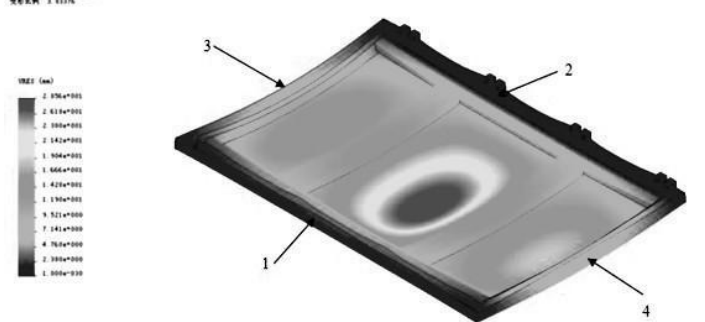

Figure 6. The deformation results of circuit breaker chamber door

\section{THE BODY STRUCTURE IMPROVEMENT OF THE SWITCHGEAR}

A. The improvement of the circuit breaker chamber's door plank

In the process of switchgear internal arc, the displacement deformation of the panel upper and lower side can't meet the safety requirements, so the gas may emit outside easily. As a result, it is necessary to improve the structural strength.Respectively fixing four bolts and hinges on both side of door plank and installing two magnitude $8.8 \mathrm{M} 8$ bolts on the upper and lower side of the door plank, changing the layout of the stiffener at the same time $^{[8]}$, as is shown in Figure 7.

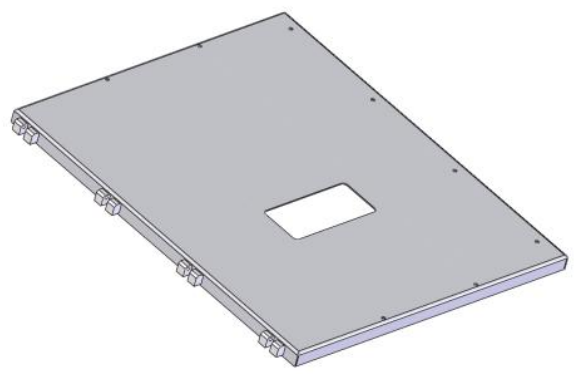

a. Positive side

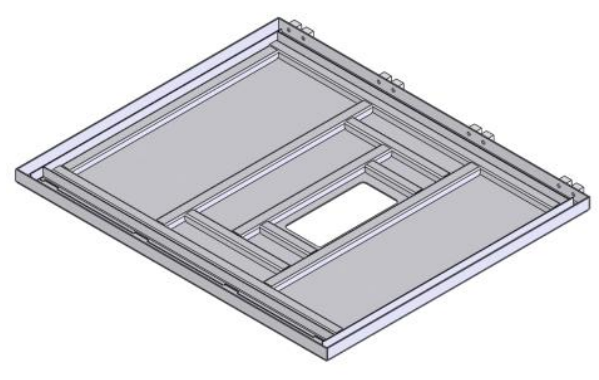

b. Negative side

Figure 7. The improved door plank structure of circuit breaker chamber

Choose Solid 10 node 187 unit type and accept the unit measuring $10 \mathrm{~mm}$ uniform grid density, as shown in figure 8.Eight threaded hole of door plank and eight hinge holes 
are completely fixed constraint, exert load type is surface load and the size is 177400 pa. Figure 9 shows that the maximal displacement deformation of the improved circuit breaker chamber door shrink to $13.35 \mathrm{~cm}$, the right side 1 , displacement deformation of left side 2 , upper side 3 , and lower side 4 are all within the scope of safety scope, guaranteeing the joint of the door plank and the pillar, so the high temperature and pressure gas will not emit from the sides of the circuit breaker chamber door plank, complying with the design requirements.

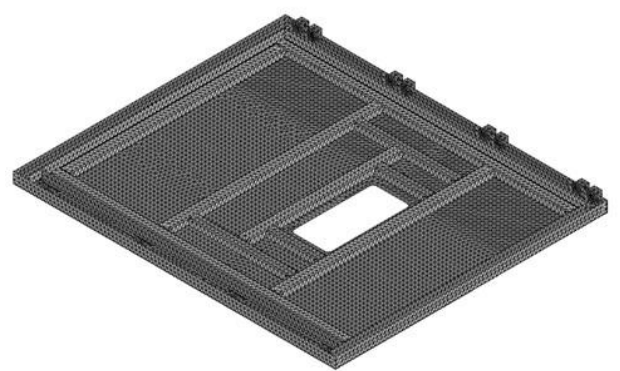

Figure 8. The improved mesh generation of improved circuit breaker chamber's door

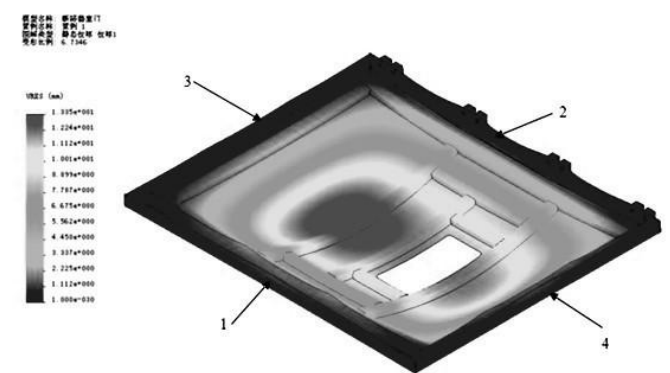

Figure 9. The deformation results of circuit breaker chamber's door

\section{B. The improvement of the cable room's door plank}

Large displacement deformation also happened at the upper and lower side of the cable chamber door in the process of internal arc, gas spewed out easily and injury the operator, therefore we need to improve the structure strength. Respectively fixing four bolts and hinges on the right and left side of door plank and installing another two bolts on the upper and lower side of the door plank, as is shown in Figure 10.

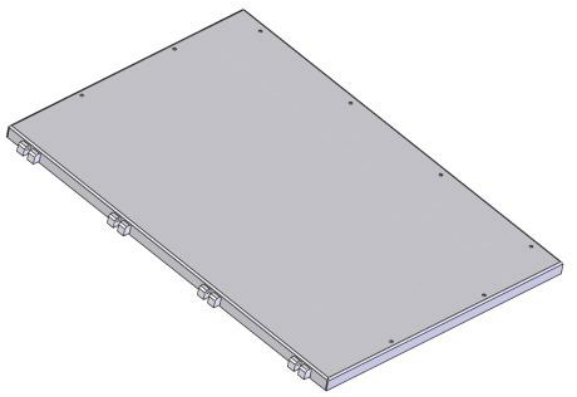

a. Positive side

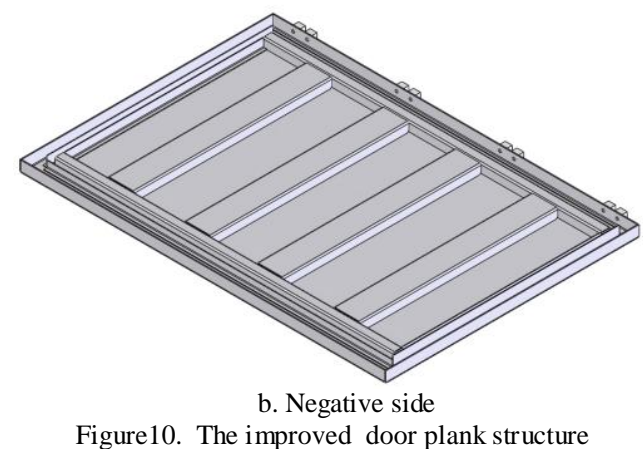

Figure10. The improved door plank structure of the cable room

Choose Solid 10 node 187 unit type and accept the unit measuring $10 \mathrm{~mm}$ uniform grid density, as shown in figure 11.Eight threaded hole of door plank and eight hinge holes are completely fixed constraint, exert load type is surface load and the size is 223700 pa. Figure 12 shows that the maximal displacement deformation of the improved circuit breaker chamber door shrink to $7.928 \mathrm{~cm}$, the right side 1 , displacement deformation of left side 2 , upper side 3 , and lower side 4 are all within the scope of safety scope, guaranteeing the joint of the door plank and the pillar, so the high temperature and pressure gas will not emit from the sides of the circuit breaker chamber door plank, complying with the design requirements.

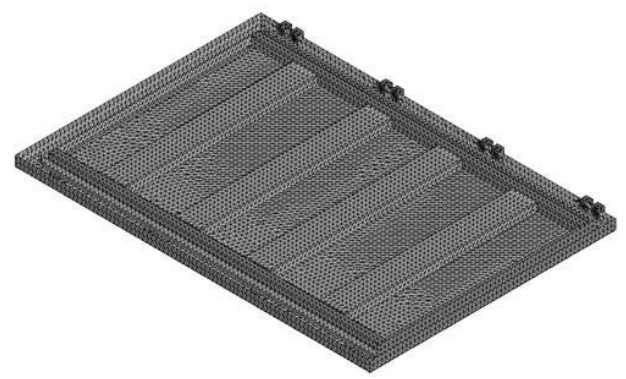

Figure 11. The improved mesh generation of improved cable roomr's door

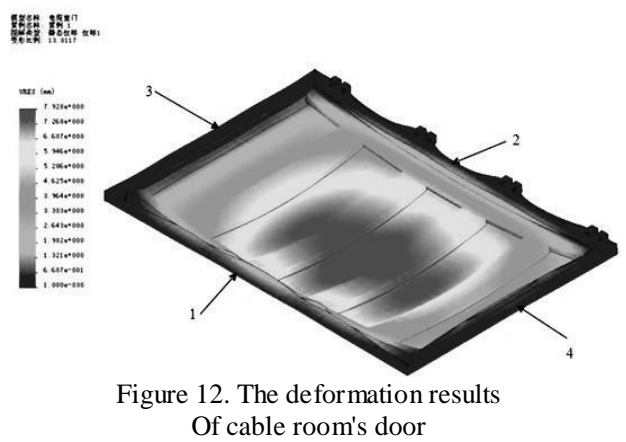

C. The improvement of the switchgear's overall structure

1)The improvement of the circuit breaker's observation window. The observation window in front of the switchgear is another weak link when the switchgear internal arc fault happens. Generally, the body accepts the ordinary glass bonding material type, this is simply not enough to resist the impact of the large parameter internal arc fault. Use $10 \mathrm{~mm}$ tempered glass, after all glass 
strength is limited, so transform the observation window from a large hole into a dense hole orifice but toughened form, to reduce the stress of the glass, ensuring the observation window can withstand the impact and won't wound the operator when the internal arc fault occurs.

2)The improvement of the metal clapboards. Strengthen the structure of the metal clapboards between each body's the compartment, and adding the right amount of $2 \mathrm{~mm}$ thickness of stiffener in both right and left side of the breaker room,the cable rooms and bus room, reducing the impact of high temperature and high pressure to the body in the maximal degree.

3)The improvement of sealing strip and the structure of the arc area. The body's front doors are all equipped with sealing strip, the compression quantity of standard of sealing strip is small, so use the sealing strip of larger compression quantity, and the back door also encryption seals, to prevent the high temperature gas from emitting outside. In order to resist the ablation of arc, the cable room, bus chamber and circuit breaker room which are close to the charged body adopt the double-layer structure, to delay the time of arc burning through. Figure 13 shows the overall structure of optimized improvement design of KYN28 switchgear $^{[9]}$.

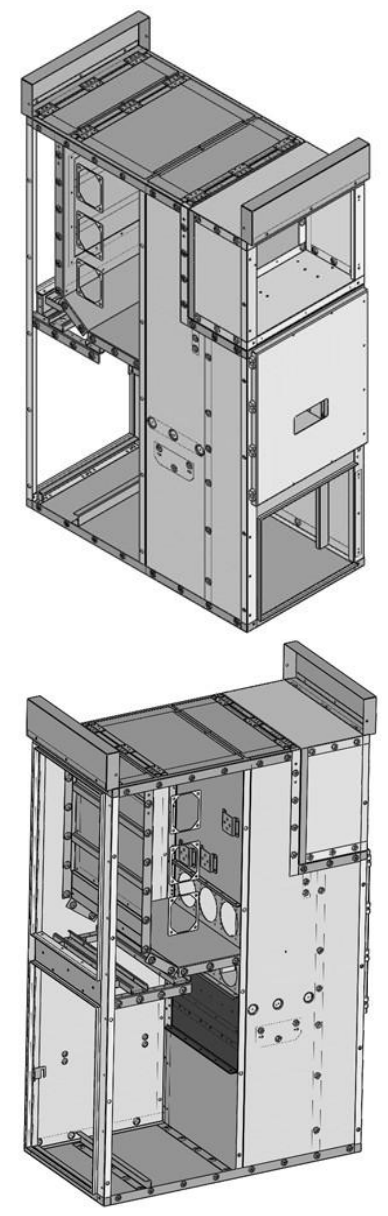

Figure 13. The overall structure of optimized improvement design of KYN28 switchgear.

\section{CONCLUSION}

According to the displacement analysis of the situation, improve the strength of swithgear's body. The purpose of improvement is to make the panel uniform stress distribution and displacement deformation within the scope of the definition, guaranteeing the panel will not be torn and four sides of cupboard's door won't spit out hot gases and scattered broken parts ${ }^{[10]}$, ensuring that the body can tolerate $1 \mathrm{~s}$ internal arc fault, providing theoretical basis for the physical design of switchgear.

\section{REFERENCES}

[1] bo-fang zhu. Principle and application of the finite element method [M]. Beijing: China water conservancy and hydropower press, 2000.

[2] Cai Bin, Chen Degui, Li Zhipeng. Simulation and Experiments on Internal Arcing Faults in MV Metal-clad Switchgear. TRANSACTIONS OF CHINA ELECTROTECHNICAL SOCIETY, 2004, 19(3): 82-87.

[3] Zhaojia Li. Development situation and development trend of switch equipment in China $[\mathrm{J}]$. Journal of guangdong electric power, 2003, 16 (1) : 1-9.

[4] shu-min zhao, wei-lin wu. Building distribution systems arc fault simulation and detection $[\mathrm{J}]$. Journal of low voltage apparatus, 2007 (4) : 38-4

[5] zhang ping. The development of the high voltage switch cabinet and present situation analysis $[\mathrm{J}]$. Journal of information science and technology of China, 2007 (24) : 89-92.

[6] Cai Bin de-gui Chen. The maximum impact load switch cabinet tolerance impact dynamics study [J]. Proceedings of the csee, 2005 (4) : 124-130

[7] Decisive Security within Milli-Seconds. Klockner-Mocller 4/96, W46735G

[8] Gregory G.D, Kon Wong, Dvorak R.F. More about arc-fault circuit interrupters [J]. IEEE Transactions on Industry Applications, 2004, 40(4): 1006-1011.

[9] S.Jamil, R.A.Jones, L.B.McClung. Arc and flash burn hazards at various levels of an electrical system[J]. IEEE Trans.Ind. Applicat, Vol. 33, p.359 366, Mar.Apr. 1997.

[10] Wang, Cao Lu, yan yangon. Aviation ac arc fault characteristics research [J]. Journal of low voltage apparatus, 2011 (2) : 19-23. 